\title{
Meningocele sacro anterior oculto
}

\author{
A. Antuña-Ramos; G. García-Fructuoso*; M. Alamar-Abril*; A. Guillén-Quesada* y J.M. Costa-Clara*
}

Hospital Universitario Central de Asturias. Oviedo. Hospital San Joan de Deu*. Barcelona.

\section{Resumen}

Introducción. El meningocele sacro anterior es una rara forma de disrafismo espinal, localizado en el espacio presacro extraperitoneal. Generalmente es asintomático y suele presentarse como una pequeña masa pélvica.

Caso clínico. Presentamos el caso de un niño de $\mathbf{1 0}$ años de edad que desarrolla una meningitis bacteriana consecuencia de un absceso pararectal que se comunica con una cavidad meningocélica sacra anterior. Recibe tratamiento quirúrgico realizándose con éxito un abordaje posterior.

Conclusión. La finalidad de la cirugía es reparar el defecto dural y obliterar la comunicación existente entre el saco tecal y el defecto herniario. El abordaje posterior es la técnica quirúrgica más efectiva puesto que tiene menor riesgo de daño neurológico que el abordaje anterior.

PALABRAS CLAVE. Meningocele sacro anterior. Masa pélvica. Absceso pararectal. Defecto dural.

\section{Occult anterior sacral meningocele}

\section{Summary}

Background. The anterior sacral meningocele is a rare form of spinal dysraphism located in the presacral extraperitoneal space. It is generally asymptomatic and appears as a small pelvic mass.

Case description. We present a case of a $\mathbf{1 0}$ year-old male that developes a bacteria meningitis because of a pararectal abscess that is connected with an anterior sacral meningocele. We successfully treated it using a posterior approach.

Conclusion. The aim of the surgery is to repair the dural defect and to obliterate the communication between the thecal sac and the herniation defect. The

Recibido: 27-05 2010. Aceptado: 5-10-10 posterior approach is the simplest and most effective surgical technique because the lower risk of neurological injury than the anterior approach.

KEY WORDS. Anterior sacral meningocele. Pelvic mass. Pararectal abscess. Dural defect.

\section{Introducción}

El meningocele sacro anterior es un raro disrafismo espinal que se origina por una herniación del saco dural a través de un defecto óseo en la pared sacra anterior. Están descritos menos de 200 casos en la literatura desde que se describiera el primero en $1837^{3}$. Se localizan en el espacio presacro extraperitoneal ${ }^{7}$. La pared del saco herniario está constituida externamente por membrana dural e internamente por membrana aracnoidea ${ }^{10}$, y en su interior contiene LCR y ocasionalmente estructuras nerviosas ${ }^{4,10}$.

La mayoría se presentan como pequeñas masas pélvicas. Suelen ser asintomáticos, sobre todo si son menores de $5 \mathrm{~cm}$ de diámetro ${ }^{10}$. Cuando dan síntomas, los pacientes generalmente presentan alteraciones urinarias o estreñimiento crónico en relación con la masa pélvica ${ }^{7}$.

La médula y el cono medular en pacientes con MSA son generalmente normales ${ }^{3}$. No suele asociarse a malformaciones cutáneas aunque sí se ha encontrado asociación con algunos tumores como quistes dermoides, teratomas sacrococcigeos y lipomas ${ }^{9,11}$. Igualmente pueden asociarse anomalías anorrectales tipo la atresia o la estenosis anal ${ }^{11}$ como ocurre en el síndrome de Currarino, que se trata de una de una enfermedad hereditaria que engloba la triada de defecto del hueso sacro, malformaciones anorrectales y presencia de una masa pélvica $^{6}$. En la mayoría de los casos dicha masa consiste en un teratoma, un MSA o la combinación de ambos.

\section{Caso clínico}

Presentamos el caso de un niño de 10 años con antece-

Abreviaturas, LCR: líquido cefalorraquídeo. MSA: meningocele sacro anterior. 


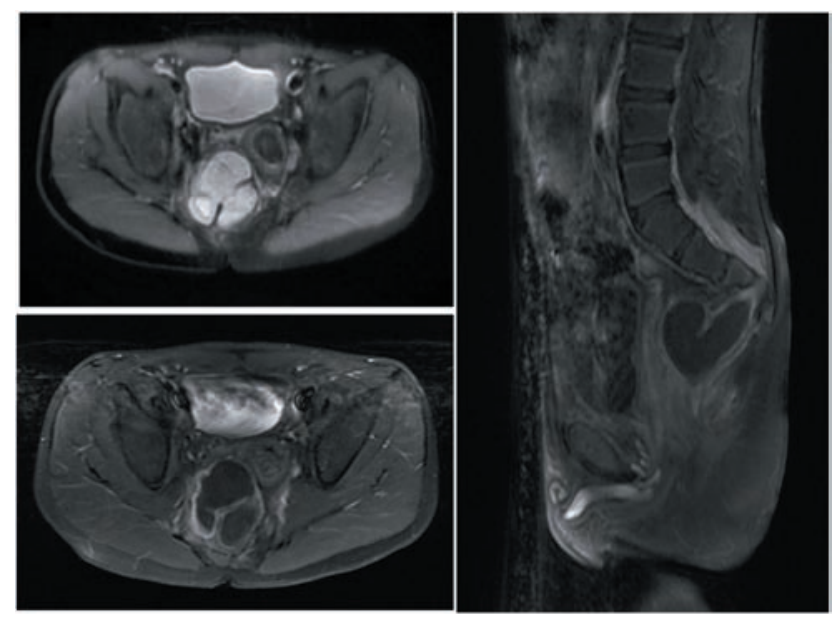

Figura 1. RM al diagnóstico inicial donde se observa la cavidad quística del meningocele sacro anterior y el absceso perirectal.

dentes de una cardiopatía compleja intervenida, riñón derecho multiquístico, vejiga disfuncional e hipoplasia sacrococcígea. A los seis meses de haber sufrido una caída golpeándose en la región glútea derecha, desarrolla un cuadro de coccigodinia que inicialmente cede con analgesia pero que dos semanas más tarde se complica con signos inflamatorios locales, compatible con absceso a ese nivel pararrectal.

Se realiza una resonancia magnética en la que se observa un cono medular de implantación baja, con agenesia de coxis; la cavidad ependimaria se ensancha distalmente y se prolonga formando un saco meníngeo en la pelvis menor, próximo el recto y al ano, compatible con meningocele sacro anterior. Existe captación de contraste en torno al cono medular, recto sigma hasta el ano, periné y zonas vecinas, que indican proceso infeccioso (Figura 1). Se decide iniciar tratamiento médico con gran mejoría clínica, encontrándose el niño asintomático a los pocos días. El paciente es dado de alta, optándose por cirugía diferida. A los quince días reingresa por aparición de fiebre y de nuevo signos inflamatorios en región glútea derecha, por lo que debe reiniciarse antibioterapia intravenosa. Durante dicho ingreso, el paciente desarrolla una meningitis bacteriana que precisa modificar y prolongar el tratamiento antibiótico.

El paciente evoluciona favorablemente (Figura 2) y, una vez finalizado el tratamiento médico, se decide abordar quirúrgicamente el meningocele. Bajo monitorización neurofisiológica y mediante un abordaje posterior, se localiza la región del defecto. En la disección se observa una estructura tubular fibrosa que comunica el canal intramedular con el saco dural anterior. Se abre el cuello de dicha malformación, extrayéndose $5 \mathrm{cc}$ de material purulento acumulado en la cavidad pélvica. Posteriormente se secciona el cuello del defecto dural, quedando completamente aislados el meningocele anterior del canal medular.
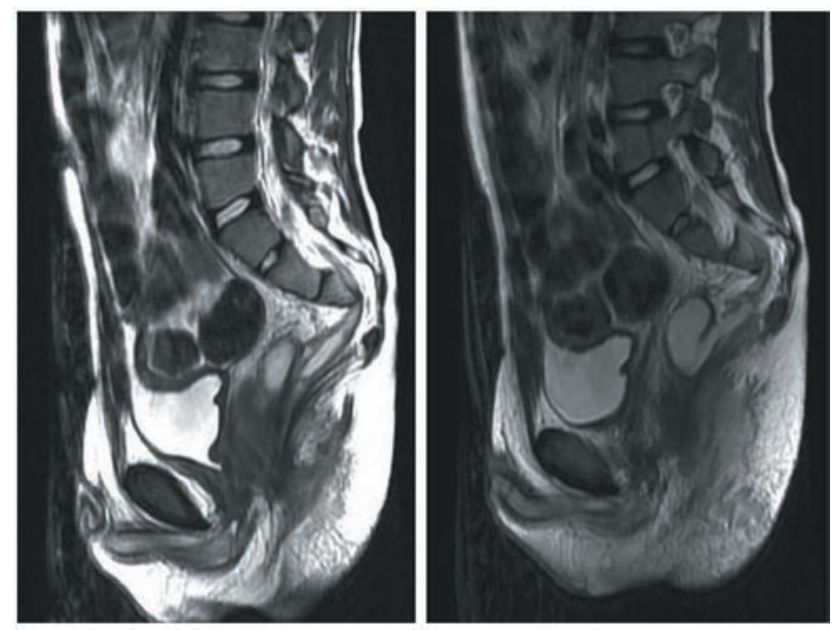

Figura 2. RM preoperatoria en la que se observa el tracto que une el saco herniario anterior con el canal medular.
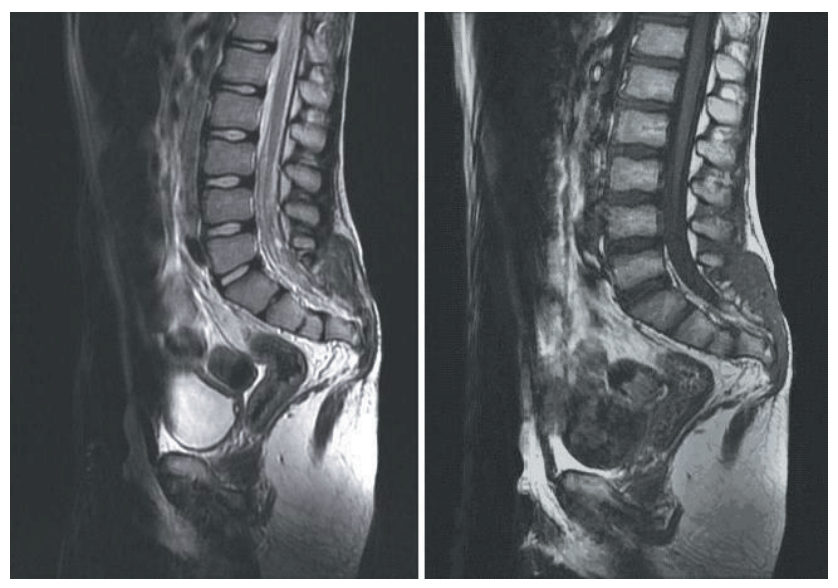

Figura 3. RM de control transcurridos 6 meses de la cirugía.

El postoperatorio transcurre sin incidencias, continuando unas semanas más con antibioterapia. Tras confirmarse radiológicamente la correcta resolución del meningocele, se decide alta domiciliaria con seguimiento ambulatorio.

Transcurrido un año, el paciente se encuentra asintomático. El absceso perianal fistulizó de manera espontánea quedando únicamente una pequeña zona de adiponecrosis en la región glútea derecha. La última RM muestra una desaparición del absceso perirectal y leve captación sólo en partes blandas, sin imágenes compatibles con persistencia del meningocele (Figura 3)

\section{Discusión}

El meningocele sacro anterior es una herniación del saco dural a través de un defecto de la superficie anterior del sacro ${ }^{10}$. Se trata de una malformación congénita muy 
poco común, que resulta de un defecto en el desarrollo de la masa celular caudal fetal, por lo que frecuentemente se asocia con otras anomalías urogenitales o rectales ${ }^{1}$ como es el síndrome de Currarino ${ }^{3,8}$. También está descrita la asociación entre meningocele sacro anterior y síndrome de Marfan ${ }^{3}$.

La etiología de los meningoceles sacro anteriores no está clara aún. Se cree que la continua pulsación del líquido cefalorraquídeo sobre la ectasia dural amplía el defecto congénito hasta provocar la herniación a través del defecto óseo constituyéndose el meningocele ${ }^{3}$.

En ausencia de anomalías urogenitales o rectales, los síntomas de MSA suelen ser muy sutiles: los pacientes suelen presentar meningitis recurrentes ${ }^{7}$, cefalea con la defecación, con los Valsalvas o con los cambios posturales, dolor lumbar, etc., y generalmente estos síndromes suelen ser debidos al efecto masa ${ }^{8}$. El quiste tiende a aumentar con la edad provocando síntomas como retenciones urinarias frecuentes, estreñimiento crónico o malestar abdominal ${ }^{7}$. Rara vez da clínica de afectación radicular ${ }^{8}$.

$\mathrm{Su}$ diagnóstico suele ser generalmente un hallazgo casual al realizar estudios de imagen. La mielografía podría ser útil para ver posibles trayectos fistulosos. La radiografía lumbosacra suele mostrar deformidades en el sacro ${ }^{7}$. Con la RM, prueba de imagen definitiva de cara a planear la cirugía ${ }^{9}$, podemos detectar si existen posibles anomalías asociadas como lipomas, senos dermoides, médula anclada $\mathrm{u}$ otros defectos del tubo neural ${ }^{8}$.

La mayoría de los autores recomiendan tratamiento quirúrgico del MSA, especialmente cuando es sintomático, por su potencial morbilidad ${ }^{2}$ y puesto que no existe posibilidad de regresión espontánea ${ }^{3,5}$. El objetivo de la cirugía es reparar el defecto dural y cerrar la comunicación entre el saco tecal y el defecto herniario ${ }^{7}$. El abordaje más aceptado es por vía posterior, porque disminuye el riesgo de lesiones neurológicas ${ }^{7,8}$ con respecto al abordaje anterior, generalmente asistido por endoscopia ${ }^{3}$. Se realiza una laminectomía y se secciona el cuello del defecto para aislarlo del espacio dural.

\section{Bibliografia}

1. Albright, A.L., Pollack, I.F., Adelson, P.D.: Principles and Practice of Pediatric Neurosurgery. New York. Thieme (eds), 1999; pp. 271-290.
2. Andersen, C., Tange, M., Bjerre, P.: Anterior sacral meningocele ocurring in one family. An authosomical dominantly inherited condition. Br J Neurosurg. 1990; 4: 59-62.

3. Ashley, W.W., Wrihgt, N.M.: Resection of a giant anterior sacral meningocele via an anterior approach: case report and review of literature. Surg Neurolog. 2006; 66: 89-93.

4. Barter, J.F., Addison, W.A., Rosenberg, E.R.: Anterior sacral meningocele presenting as a pelvis mass and diagnosed onlyat celiotomy alteran extensiv workup: a case report. J Reprod Med. 1983; 28: 684-686.

5. Brem, H., Baewere, B.L., Colombquani, P.M.: Neonatal diagnosis of a presacral mass in the presence of congenital anal stenosis and partialsacral agenesis. J Ped Surg. 1989; 24: 1076-1078.

6. Emans, P.J., Aalst, J., Heurn, E.L.W., Marcelis, C.L.M., Kootstra, G., Beets-Tan, R.G., Vles, J.S., Beuls, E.A.: The Currarino trial: neurosurgical considerations. Neurosurgery. 2006; 58: 924-929.

7. Fitzpatrick, M., Taylor, W.A.: Anterior sacral meningocele associated with a rectal fistula. Case report and review of the literature. J Neurosurg (Spine 1)1999; 91: 124-127.

8. Gardner, P.A., Albright, A.L.: "Like mother, like son": Hereditary anterior sacral meningocele. J Neurosurg. 2006; 104: 130-142.

9. Quigley, M.R., Schinco, F., Brown, J.T.: Anterior sacral meningocele with an unusual presentation. Case report. J Neurosurg. 1984; 61: 790-792.

10. Santana, J.M., Gómez, A., Alemán, P., Travieso, MM.: Ureterohidornefrosis severa asociada a meningocele sacro anterior gigante asintomático: presentación de un caso y revisión de la literatura. Radiología. 2007 49: 43-46.

11. Villarejo, F., Scavone, C., Blazquez M.G., PascualCastroviejo, I., Perez-Higueras, A., Fernandez-Sanchez, A., Garcia Bertrand, C.: Anterior sacral meningocele: review of literature. Surg Neurol. 1983; 19: 57-71.

Antuña-Ramos, A.; García-Fructuoso, G.; Alamar-Abril, M.; Guillén-Quesada, A.; Costa-Clara, J.M.: Meningocele sacro anterior oculto. Neurocirugía 2011; 22: 342-346.

Correspondencia: Aida Antuña Ramos M.D. Hospital Universitario Central de Asturias. Fernández Ladreda, 30, 6º B, C.P.: 33011, Oviedo (Asturias). España.

Email: aida_ar20@hotmail.com 


\section{Comentario al trabajo Meningocele sacro anterior oculto de Antuña Ramos y col.}

La triada o síndrome de Currarino es una malformación hereditaria infrecuente de la región caudal consistente en un defecto óseo del sacro que se acompaña de malformaciones anorrectales y una masa presacra. Se transmite de forma autosómica dominante con una expresión fenotípica muy variable, lo que supone que algunos individuos presenten la forma del síndrome completo, mientras que otros pacientes puedan estar afectados de un meningocele anterior sacro sin problemas anorrectales. Según esta definición, el caso que presentan Antuña y cols. podría corresponder a una forma incompleta de síndrome de Currarino. El trastorno genético que lo origina se localiza en la región 7q36, que contiene el gen homebox XLXB9 ${ }^{3,4}$. Esta alteración se relacionaría con un fallo en la separación dorsoventral de la eminencia caudal del endodermo del intestino posterior. Esto alteraría de forma secundaria el desarrollo de la cloaca y el sacro, dando como resultado malformaciones anorrectales y genitourinarias, defectos óseos en el sacro y la presencia de masas presacras que completan la triada. Entre éstas últimas el meningocele anterior es la más frecuente $(60 \%$ de los casos) seguida del teratoma maduro (25\%) y otros tumores $(15 \%)^{2}$. La disrupción de la neurulación secundaria explicaría la presencia de un disrrafismo espinal oculto y la médula anclada en algunos casos.

La presentación clínica del síndrome de Currarino oscila desde individuos asintomáticos, con diagnóstico incidental de un meningocele anterior sacro sin otras malformaciones hasta meningitis debidas a fístulas entre el intestino y el saco tecal (como es el caso que presentan los autores) o graves alteraciones anorrectales que incluyen afectaciones motoras de la función esfinteriana. El síntoma más frecuente es el estreñimiento crónico que se ha relacionado con las estenosis rectales, disfunciones esfinterianas o con el efecto de masa de la tumoración presacra ${ }^{1}$. Hasta un $18 \%$ de los casos pueden presentar médula anclada, como en el caso que nos ocupa. Las lesiones presacras más frecuentes son el meningocele anterior sacro o el teratoma, aunque se han descrito también tumores dermoides, lipomas o quistes neuroentéricos. Pueden asociarse también otras anomalías como duplicidades del tracto urogenital (útero bicorne, uréteres dobles,), diversos tipos de fístulas, cono anclado, vejiga neurógena y siringomielia.

El tratamiento quirúrgico es la alternativa más habitual si bien en determinados casos sin tumor presacro asociado y meningoceles pequeños con diagnóstico incidental puede contemplarse un tratamiento expectante. La recomendación de cirugía se debe principalmente al riesgo de complicaciones infecciosas (meningitis por enterobacterias) debido a la continuidad del saco con la cara posterior del recto y sigma. Se han descrito también roturas espontáneas del saco tecal y degeneraciones malignas del teratoma asociado $^{6}$. Entre las distintas posibilidades de tratamiento quirúrgico los autores comentan las ventajas de un abordaje posterior respecto a la vía anterior. Indudablemente, la vía sagital posterior presenta la posibilidad de controlar, manipulando directamente, las estructuras del sistema nervioso (cono medular, raíces sacras y dermoides intradurales) y tratar las malformaciones asociadas a una posible médula anclada. Se trata además de un campo más habitual para el neurocirujano y permite explorar y ligar el cuello del saco tecal herniado, tanto por vía intradural como de forma extradural. Las relaciones anatómicas del saco tecal con las estructuras de la pelvis menor, fundamentalmente la cara posterior del recto, dificultan su disección manteniendo la integridad de la duramadre herniada, que generalmente es más delgada y atrófica. Sin embargo, en los casos de una tumoración presacra voluminosa, como pudiera ser un teratoma o un dermoide con extensión hacia el promontorio, no debe desestimarse esta vía ya que es la única forma de controlar el tumor pélvico. La extirpación en dos tiempos quirúrgicos (uno anterior y otro posterior) es también una opción. En los casos de síndrome de Currarino con tumoración presacra pero sin meningocele asociado se ha descrito un abordaje sagital posterior trans-esfinteriano, que a través del rafe medio perineal, de forma transrectal y preservando la función esfinteriana anal, permite acceder a la región presacra y extirpar teratomas y tumores dermoides asociados a esta entidad ${ }^{5}$.

El caso presentado por Antuña y cols. demuestra la necesidad de llevar a cabo un tratamiento precoz tan pronto se llegue al diagnóstico de la malformación que nos ocupa. Hemos tenido oportunidad de tratar varios casos de síndrome de Currarino y nuestra experiencia en el manejo de las malformaciones que afectan a la región caudal se ha visto beneficiada por el abordaje multidisciplinar. Las aportaciones procedentes de otros especialistas y su enfoque quirúrgico permite completar el abanico de posibilidades terapéuticas que se pueden ofertar a estos pacientes, en ocasiones muy complejos.

\section{Biliografía}

1 Cama, A., Palmieri, A., Capra, V., Piatelli, G. L., Ravegnani, M., Fondelli, P.: Multidisciplinary management of caudal regression syndrome (26 cases). Europ. J. Pediat. Surg. 1996; 6 (suppl. 1): 44-46.

2. Kochling, J., Karbasiyan, M., Reis, A.: Spectrum of mutations and genotype-phenotype analysis in Currarino syndrome. Europ. J. Hum. Genet. 2001; 9: 599-605.

3. Lynch, S. A., Wang, Y., Strachan, T., Burn, J., Lindsay, 
S.: Autosomal dominant sacral agenesis: Currarino syndrome. J. Med. Genet. 2000; 37: 561-566.

4. Ross, A. J., Ruiz-Perez, V., Wang, Y., Hagan, D.M., Scherer, S., Lynch, S. A., Lindsay, S., Custard, E., Belloni, E., Wilson, D. I., Wadey, R., Goodman, F., Orstavik, K. H., Monclair, T., Robson, S., Reardon, W., Burn, J., Scambler, P., Strachan, T.: A homeobox gene, HLXB9, is the major locus for dominantly inherited sacral agenesis. Nature Genet. 1998; 20 : 358-361

5. The posterior sagittal trans-sphincteric and trans-rectal approaches. Peña A, Hong A. Tech Coloproctol. 2003 Apr; 7: 35-44.

6. Urioste, M., Garcia-Andrade, M. del C., Valle, L., Robledo, M., Gonzalez-Palacios, F., Mendez, R., Ferreiros, J., Nuno, J., Benitez, J.: Malignant degeneration of presacral teratoma in the Currarino anomaly. Am. J. Med. Genet. 2004; 128A: 299-304.

J. Hinojosa A. García Vázquez

Madrid 